\title{
The Zebra in the room
}

\author{
Grace Lee
}

Published online: 3 January 2012

(C) Springer Science+Business Media, LLC 2011

Heparin induced thrombocytopenia (HIT) is an immunemediated prothrombotic disorder which classically presents with declining platelet counts 5-10 days after heparin exposure. Thrombotic complications occur in an estimated $20-50 \%$ of patients with thrombocytopenia and can affect both the venous or arterial circulation [1]. In patients with a classic presentation of thrombosis in the setting of thrombocytopenia, the diagnosis of HIT is readily made. However, in patients with complicated medical issues and atypical presentations, the diagnosis is more challenging. Rarely, HIT presents with unusual manifestations: adrenal hemorrhagic necrosis, skin necrosis at heparin injection sites, warfarin-associated venous limb gangrene, cerebral venous thrombosis, disseminated intravascular coagulation, or systemic reactions with heparin administration. Often, these unusual sequelae are not accompanied by thrombocytopenia (as with skin necrosis), or paradoxically, are associated with a hemorrhagic event (as a consequence of thrombosis) [2].

In this issue, Thota et al. describe a patient with HIT presenting with hemodynamic collapse as a result of adrenal insufficiency caused by adrenal vein thrombosis and adrenal necrosis. Although adrenal hemorrhagic necrosis is a very rare complication of HIT, the authors recognized that the patient was thrombocytopenic after receiving low-molecular-weight heparin and that adrenal hemorrhage, as a result of adrenal vein thrombosis, is frequently associated with HIT [3].

This case highlights the importance of recognizing the unusual thrombotic complications of HIT. Although the diagnosis of HIT can be difficult, recognition of the unusual sequelae of HIT allows for prompt diagnosis and initiation of therapy, with potential to prevent significant morbidity and mortality.

\section{References}

1. Arepally GM, Ortel TL (2006) Clinical practice. Heparin-induced thrombocytopenia. N Engl J Med 355:809-817

2. Warkentin TE, Greinacher A (2004) Heparin-induced thrombocytopenia, 3rd edn. Marcel Dekker, Inc, New York

3. Vella A, Nippoldt TB, Morris JC III (2001) Adrenal hemorrhage: a 25-year experience at the Mayo Clinic. Mayo Clin Proc 76:161168
G. Lee $(\bowtie)$

Duke University, DUMC 3841, Durham, NC 22710, USA

e-mail: grace.lee@duke.edu 